\title{
The magnetic field structure in the multi-source magnetized core NGC 2024 FIR 5
}

\author{
Felipe de O. Alves ${ }^{1}$, J. M. Girart ${ }^{1}$, S.-P. Lai ${ }^{2}$, \\ R. Rao $^{3}$ and Q. Zhang ${ }^{4}$ \\ ${ }^{1}$ Institut de Ciències de l'Espai (IEEC-CSIC), Bellaterra, Catalunya 08193, Spain \\ email: oliveira@ieec.uab.es \\ ${ }^{2}$ Institute of Astronomy and Department of Physics, National Tsing Hua University, \\ Hsinchu 30043, Taiwan \\ email: slai@phys.nthu.edu.tw \\ ${ }^{3}$ Institute of Astronomy and Astrophysics, Academia Sinica, P.O. Box 23-141, \\ Taipei 10617, Taiwan \\ email:rrao@sma.hawaii.edu \\ ${ }^{4}$ Harvard-Smithsonian Center for Astrophysics, 60 Garden Street, Cambridge, \\ MA 02138, USA \\ email: qzhang@cfa.harvard.edu
}

\begin{abstract}
This work reports high resolution SMA polarimetric observations toward NGC 2024 FIR 5, a magnetized core previously found to harbour protostars. Our $345 \mathrm{GHz}$ data indicates the presence of an extended dust emission associated with the dense core where the protostars are embedded. The $3 \sigma$ polarized intensity shows depolarization toward the peak of Stokes I emission. This diminishing polarized flux implies that the alignment efficiency of the core dust grains is low within higher column densities where grain properties are likely different. The derived magnetic field geometry exhibits pinched field lines which are typical in evolved supercritical clouds where the magnetic field no longer support the core from collapsing. As a consequence for protostars, the gravitational pulling along the disk's long axis makes an equatorial bend to the field lines that, in turn, results in a hourglass shape. The SMA field structure agrees perfectly with the BIMA map. However, models are still necessary to provide a complete description of the evolutionary scenario of FIR 5.
\end{abstract}

Keywords. ISM: individual (NGC 2024) - ISM: magnetic fields - polarization - stars: formation - techniques: interferometric

\section{Introduction}

Understanding the evolution of molecular clouds and protostellar cores is one of the outstanding concerns of modern astrophysics. Particularly, efforts are concentrated in determine which physical agents are mainly responsible to control the dynamical properties of dense cores. It is already accepted by the astronomical community that magnetic fields must be taken into account in evolutionary models of collapsing protostellar cores (Shu, Allen, Shang, et al. (1999)). Although some theories claim that turbulent supersonic flows drives star formation in the interstellar medium (Elmegreen \& Scalo (2004), Mac Low \& Klessen (2004)), some new results demonstrate that the ambipolar diffusion collapse theory reproduces properly observed molecular cloud lifetimes and star formation timescales (Tassis \& Mouschovias (2004), Mouschovias, Tassis \& Kunz (2006)).

Recent advances in instrumentation for astronomical polarimetry improved significantly the techniques to measure the Galactic magnetic field. Several telescopes and 
interferometers are now capable of doing polarimetric observations at distinct wavebands. At $\mathrm{mm} / \mathrm{submm}$ wavelengths, the Sub-millimeter Array (Hawaii) is the only instrument that allows to observe the polarization flux at high sensitivity and at the same time high angular and spectral resolution.

\section{The Sub-millimeter Array}

\subsection{SMA capabilities}

The Sub-millimeter Array is installed at the Mauna Kea summit, in Hawaii. Its superior site results in a very good atmospheric transmissivity for sub-millimeter waves. SMA has a bandwidth correlator composed by 2 sidebands with a width of $2 \mathrm{GHz}$ each one. As a consequence, the $\mathrm{mm} / \mathrm{submm}$ observations increase sensitivity in detection of thermal emission (from approximately 10 to a few hundred $\mathrm{K}$ ) from dust and cool gas in molecular clouds, dense cores and stellar envelopes.

The instrument was designed to achieve sub-arcsecond spatial resolutions, reaching values as high as $0.15^{\prime \prime}$ when operating at the highest frequencies in the very extended configuration. The high resolution of the SMA makes possible to trace the thermal emission of dust grains at physical scales of $10^{2}$ astronomical units (for objects closer than 1 $\mathrm{kpc}$ ) and, therefore, is able to resolve compact dust cores into multiple components. For a detailed description of SMA, please refer to Ho, Moran \& Lo (2004).

\subsection{Polarization with SMA}

Assuming that dust grains are aligned perpendicularly with respect to the interstellar magnetic field, the thermal emission detected by the telescopes must have a degree of polarization. Cold dust emits mainly at far-IR, sub-mm and $\mathrm{mm}$, optically thin wavelengths not affected by scattering or absorption. As described in the previous section, SMA has the suitable instrumentation for observations at sub-mm and $\mathrm{mm}$ bands. In general, the polarized flux of a source is only a small fraction of the total emission, but the high sensitivity of SMA represents an improvement in polarimetric observations compared to other interferometers (e.g. OVRO, BIMA).

SMA receivers at $345 \mathrm{GHz}$ are single and linearly polarized. By using a quarter wave plate attached to each receiver, incoming radiation is converted to circular polarization $(\mathrm{L}, \mathrm{R})$. The SMA correlator combines the circular polarization vectors (RR, LL, RL, LR) into the Stokes parameters needed to calculate the polarization fraction and the position angle of the polarization vectors. A complete description of the polarimetric system of SMA can be found in Marrone \& Rao (2008).

\subsection{Science with SMA: first results in polarization}

Distinct classes of protostellar cores were already observed with SMA in mode of polarization. Recently, the polarimetric properties of G5.89-0.39, a massive ultra compact HII region, were obtained with SMA at high angular resolution $\left(3^{\prime \prime}\right)$ (Tang, Ho, Girart, et al. (2008)). The extended polarized flux detected around the ionized gas is related to the kinematics of the region, where the magnetic field morphology seems to be disturbed by the expansion of the HiI region and outflows produced by young stellar objects in G5.89.

The textbook case of a pinched magnetic field of a low mass young stellar system is NGC 1333 IRAS 4A (Girart, Rao \& Marrone (2006)). With a resolution of $1.6^{\prime \prime} \times 1.0^{\prime \prime}$, the dust emission traces a physical scale of 300-1000 AU's, associated to a circumbinary envelope. The magnetic field strength for this source was estimated in $5 \mathrm{mG}$ and the derived mass-to-flux ratio was 2 , indicating that the core is collapsing. Such supercritical 
stage is reflected in the SMA polarization maps (Fig. 1), which indicates a clear hourglass morphology for the plane-of-sky magnetic field, in agreement with theories of collapse of magnetized clouds (Fiedler \& Mouschovias (1993), Galli \& Shu (1993)). Recently, these data was used to test models of collapsing magnetized cores from Galli \& Shu (1993) and from Shu, Galli, Lizano, et al. (2006). The agreement between the SMA data and the models leads to the assumption that field dissipation must have occurred in the central parts of IRAS 4A (Gonçalves, Galli \& Girart (2008)). This could explain the observed fragmentation (Reipurth, Rodríguez, Anglada, et al. (2002), Looney, Mundy \& Welch (2000)) that may be occurring in this core, since magnetic braking would be alleviated.

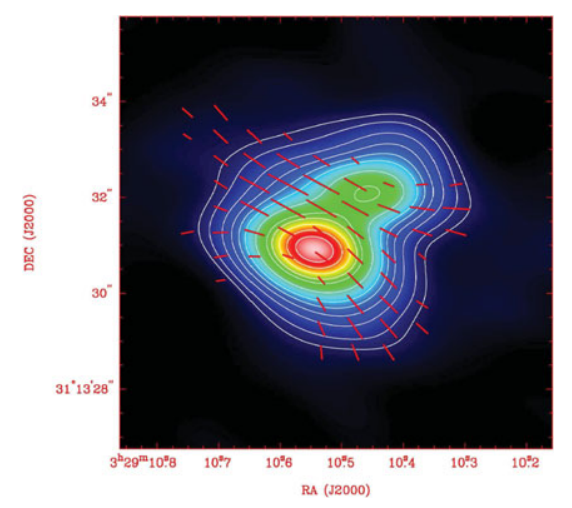

Figure 1. SMA dust emission polarization maps of low mass protostars: NGC 1333 IRAS 4A. Contours and color scale refers to the $870 \mu \mathrm{m}$ dust continuum emission. The emission is resolved into a binary system (Girart, Rao \& Marrone (2006)).

\section{NGC 2024: polarization observations}

NGC 2024 is a massive star forming region at 415 pc from the Sun, in the Orion B giant molecular cloud. The region contains a luminous His region where several reflection nebulae are found. Also a north-south molecular ridge is seen toward its center and corresponds to a dust lane in the optical image. Along this ridge, several dust cores were detected and catalogued at 1300 and $350 \mu \mathrm{m}$ (Mezger, Chini, Kreysa, et al. (1988), Mezger et al. (1992)). These cores were assigned as FIR (from Far-InfraRed) cores and, in this work, we study the brightest and most evolved of them, FIR 5. A highly collimated outflow extended $\sim 5^{\prime}$ south of the core (Richer, Hills \& Padman (1992)) is associated to this core which has an intermediate mass and multiple components (Wiesemeyer, Güsten, Wink, et al. (1997)).

Near-infrared imaging polarimetry was conducted by Kandori, Tamura, Kusakabe, et al. (2007) toward NGC 2024. They found a prominent and extended polarized nebula over NGC 2024 and constrained the location of the illuminating source through an analysis of polarization vectors (Fig. 2). A massive star, IRS 2b, with spectral type of O8-B2, is located at the center of the centrosymmetric vector pattern observed in $J, H$ and $K$.

\subsection{BIMA polarization maps of FIR 5}

The first results on the polarized thermal emission from FIR 5 was done by Lai, Crutcher, Girart, et al. (2002). These authors obtained BIMA dust continuum polarization maps combining three different configurations. With the highest resolution ever obtained 


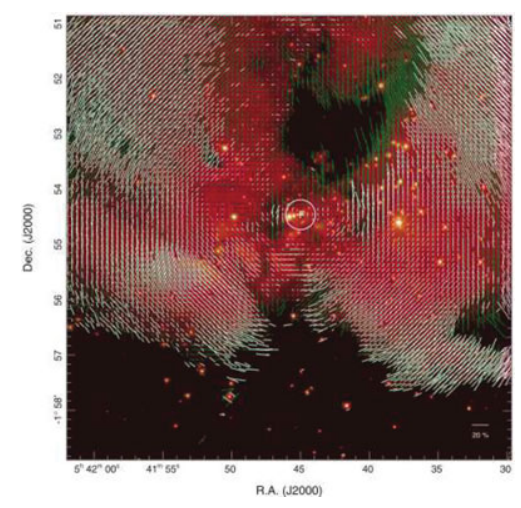

Figure 2. Polarization generated by scattering toward NGC 2024. Vectors exhibits a centrosymmetric symmetry. Normal lines of each vector intersect at a position near the center of the cloud $\left(\alpha=54^{\mathrm{h}} 41^{\mathrm{m}} 45.01^{\mathrm{s}}, \delta=\right.$ $\left.-1^{\circ} 54^{\prime} 27^{\prime \prime} .7\right)$. The massive star IRS $2 \mathrm{~b}$ is probably the illuminating source of the region since its position coincides with the center of symmetry of the polarization vectors (Kandori, Tamura, Kusakabe, et al. (2007)).

$\left(1.6^{\prime \prime} \times 1.2^{\prime \prime}\right.$, P.A. $\left.=11^{\circ}\right)$, they could resolve the $1.3 \mathrm{~mm}$ emission into 7 clumps with flux peak stronger than $7 \sigma\left(1 \sigma \sim 3.3 \mathrm{mJy}_{\text {beam }}{ }^{-1}\right)$. The extended dust emission harbors four of the detected clumps, and for this reason it is supposed to represent a circumstellar disk with multiple young objects. The three remaining clumps are supposed to be collapsing toward this disk.

The polarization emission extends over an area of $\sim 8$ beam sizes along the direction perpendicular to the elongated continuum emission. Figure 3, left panel, shows polarization vectors where the observed linearly polarized intensity is greater than $3 \sigma_{I_{P}}\left(1 \sigma_{I_{P}} \sim\right.$ $2.1 \mathrm{mJy} \mathrm{beam}^{-1}$ ). The polarimetric map derived for FIR 5 was fitted to a set of parabolas with the same focus point. It means that the magnetic fields lines in the core are systematically curved, consistent with the hourglass morphology predicted by theoretical works. Although some missing polarized flux makes the hourglass shape incomplete (probably due to a lower column density to the east of the region), these authors used the Chandrasekhar-Fermi formula to estimate the magnetic field strength at the core from the fit residuals between the observed and synthetic position angles. As a result, the plane-of-sky component of the magnetic field was calculated as $\sim 2 \mathrm{mG}$. The turbulentto-magnetic energy was estimated as less than 0.14, meaning that the magnetic field likely dominates the turbulent motions in the core.

\subsection{SMA polarization maps of FIR 5}

The SMA observations of FIR 5 were carried out in the compact configuration at an LO frequency of $345 \mathrm{GHz}$. The resulting map have a spatial resolution of 2.9 " $\times 1.7$ " with a beam position angle of $-37^{\circ}$. The peak of continuum intensity is $1.3 \mathrm{Jy} / \mathrm{beam}$ and the $\mathrm{rms} \sim 15 \mathrm{mJy} /$ beam. The polarized intensity have a peak intensity of $66 \mathrm{mJy} / \mathrm{beam}$. At this position, the polarization degree reaches $\approx 19 \%$.

The resulting polarization pattern is consistent with the previous one obtained by Lai, Crutcher, Girart, et al. (2002) with BIMA, however it is more compact. In both cases, the polarized flux has a peak to the north of the continuum peak and decreases in magnitude toward it. The observed depolarization could be explained by the lower dust alignment efficiency in high density regions, although the same effect could be produced also by geometrical purposes, due simply by the projection of field lines on the line-of-sight (Gonçalves, Galli \& Walmsley (2005)). Figure 3, right panel, exhibits SMA continuum emission contours at $10 \sigma$ threshold and polarized vectors at a $3-\sigma$ level.

SMA continuum emission is elongated at the east-west direction, as found with BIMA, and it is possibly tracing a protostellar core. The main contribution of the polarized flux has a position angle perpendicular to this putative disk but, in contrast to BIMA maps, there is a larger contribution at the eastern part of the core. 

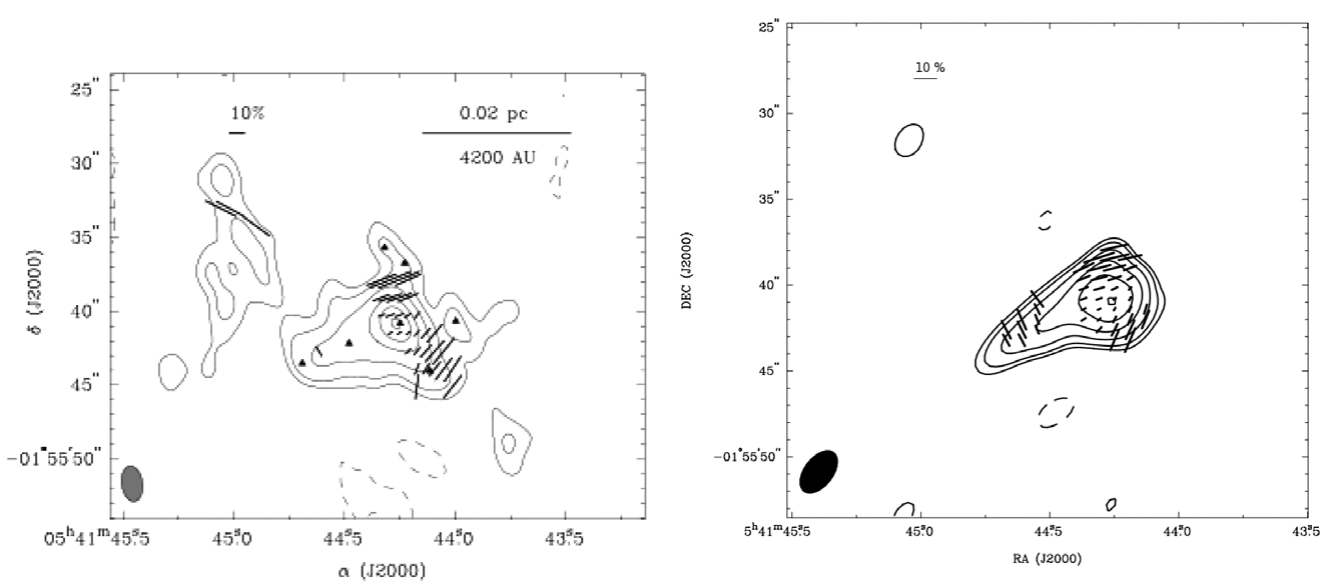

Figure 3. Dust polarization maps of NGC 2024 FIR 5. For both maps, contours are the continuum emission and the vectors refer to the polarized flux. Left panel: BIMA map obtained by Lai, Crutcher, Girart, et al. (2002). Right panel: SMA map of FIR 5. Both maps have the same global patterns, but SMA is more sensitive to the polarized flux at the east part of the core.
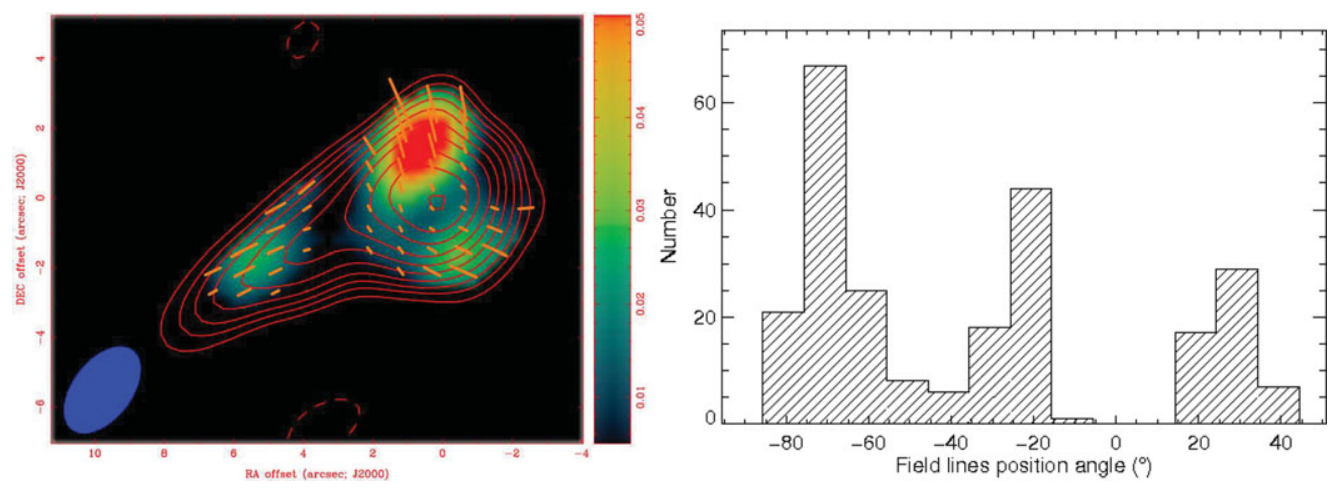

Figure 4. Left panel: magnetic field lines (yellow bars) for a $2 \sigma$ polarization intensity level. Color scale indicates de polarized flux and red contours the continuum emission. Right panel: histogram of field lines position angle.

After a $90^{\circ}$ rotation of the polarization vectors, we obtain the plane-of-sky component of the magnetic field lines toward the core. As expected, a partial hourglass field morphology is obtained at scales of $10^{3}$ AU's. An histogram of the field lines position angle (Fig. 4, right panel) reproduces partially the preferential orientations for the field lines seen in Fig. 4, left panel. There is an abrupt change (by almost $90^{\circ}$ ) in the direction of the magnetic field east of the hourglass shape.

\section{Conclusions and future work}

NGC 2024 FIR 5 has all signatures of a magnetized core under gravitational collapse driven by ambipolar diffusion. The magnetic field geometry is characterized by bended field lines likely produced by the gravitational pull along the protostellar disk, typical of collapsing cores which just achieve a supercritical regime. The magnetic field morphology observed for FIR 5 seems to be reproduced in different physical scales, what may 
suggest that the magnetic pressure during the collapse stage must be taken into account independently of the core mass.

For a better evolutionary description of FIR 5, we will apply geometrical models to fit the observed field orientation. By means of the Chandrasekhar-Fermi formula, we will use the residuals of the model to estimate the magnetic field strength and the magnetic energy to compare with the turbulent energy of the system. In the future, by use of radiative transfer models, we plan to derive the physical properties of the core and compare with observations and previous work.

\section{Acknowledgements}

We would like to thank the SMA staff for the support during observations.

\section{References}

Elmegreen, B. G. \& Scalo, J 2004, ARAA 42, 211

Fiedler, R. A. \& Mouschovias, T. Ch. 1993, ApJ 415, 680

Galli, D. \& Shu, F. H. 1993, ApJ 417, 243

Girart, J. M., Rao, R., \& Marrone, D. P. 2006, Science 313, 812

Gonçalves, J., Galli, D., \& Walmsley, M. 2005, A\&A 430, 979

Gonçalves, J., Galli, D., \& Girart, J. M. 2008, A\&A 490, L39

Ho, P. T. P., Moran, J. M., \& Lo, K. Y. 2004, ApJ 616, L1

Kandori, R., Tamura, M., Kusakabe, N., Nakajima, Y., Nagayama, T., Nagashima, C., Hashimoto, J., Ishihara, A., Nagata, T., \& Hough, J. H. 2007, PASJ 59, 487

Lai, S. -P., Crutcher, R. M., Girart, J. M., \& Rao, R. 2002, ApJ 566, 925

Looney, L. W., Mundy, L. G., \& Welch, W. J. 2000, ApJ 529, 477

Mac Low, M.-M. \& Klessen, R. S. 2004, Reviews of Modern Physics 76, 125

Marrone, D. P. \& Rao, R. 2008, in W. D. Duncan, W. S. Holland, S. Withngton \& J. Zmuidzinas (eds),Millimeter and Submillimeter Detectors and Instrumentation, SPIE 7020

Mezger, P. G., Chini, R., Kreysa, E., Wink, J. E., \& Salter, C. J. 1988, A\&\&A 191, 44

Mezger, P. G., Sievers, A. W., Haslam, C. G. T., Kreysa, E., Lemke, R., Mauersberger, R., \& Wilson, T. L. 1992, A\&BA 256, 631

Mouschovias, T. C., Tassis, K., \& Kunz, M. W. 2006, ApJ 646, 1043

Reipurth, B., Rodríguez, L. F., Anglada, G., \& Bally, J. 2002, AJ 124, 1045

Richer, J. S., Hills, R. E., \& Padman, R. 1992, MNRAS 254, 525

Shu, F. H., Allen, A., Shang, H., Ostriker, E. C., \& Li, Z. 1999, in: C. J. Lada \& N. D. Kylafis (eds.), in The Origin of Stars and Planetary Systems, p.193

Shu, F. H., Galli, D., Lizano, S., \& Cai, M. 2006, ApJ 647, 382

Tang, Y. -W., Ho, P. T. P., Girart, J. M., Rao, R., Koch, P. M., \& Lai, S. -P. 2008, ApJ, submitted

Tassis, K. \& Mouschovias, T. C. 2004, ApJ 616, 238

Wiesemeyer, H., Güsten, R., Wink, J. E., \& Yorke, H. W. 1997, A\& A 320, 287

\section{Discussion}

De Gouveia Dal Pino: In one of the binary targets you showed there was a very organized B-field structure in each of the sources. Is there any evidence for an hour-glass shape in each of them?

Alves: For the brightest source, one can see clearly the magnetic field with an hourglass morphology. The other component, that is unresolved in previous observations with JCMT, doesn't exhibit bended field lines. At higher resolutions, maybe we can detect an hour-glass morphology if this is the case of a supercritical collapsing core. 
ZINNECKER: The obvious question would be: is the NGC2024 FIR S core supercritical or subcritical? Is the core collapsing?

Alves: Yes. Previous BIMA observations resolved FIR S into 7 components by Lai et al. (2002) (These authors estimated a B strength of $2 \mathrm{mG}$ and a turbulent to magnetic energy ratio of 0.14 ). In addition, a highly collimated outflow is observed toward it. These star-forming signatures, combined with our SMA hourglass magnetic field for this core, indicate that it is collapsing and maybe stare to experience fragmentation, in agreement with modern theoretical models of core collapse including magnetic support.

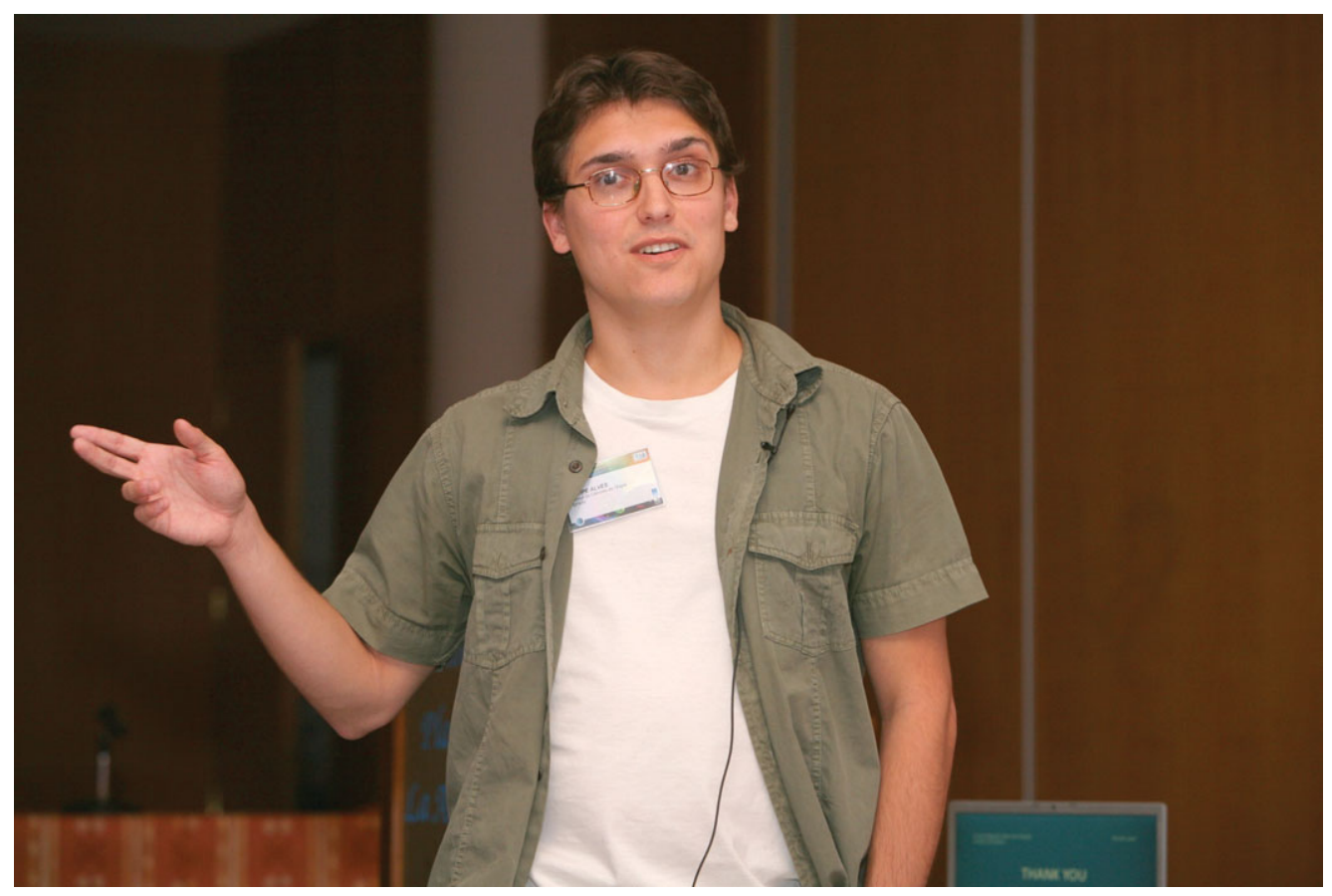

Felipe Alves 


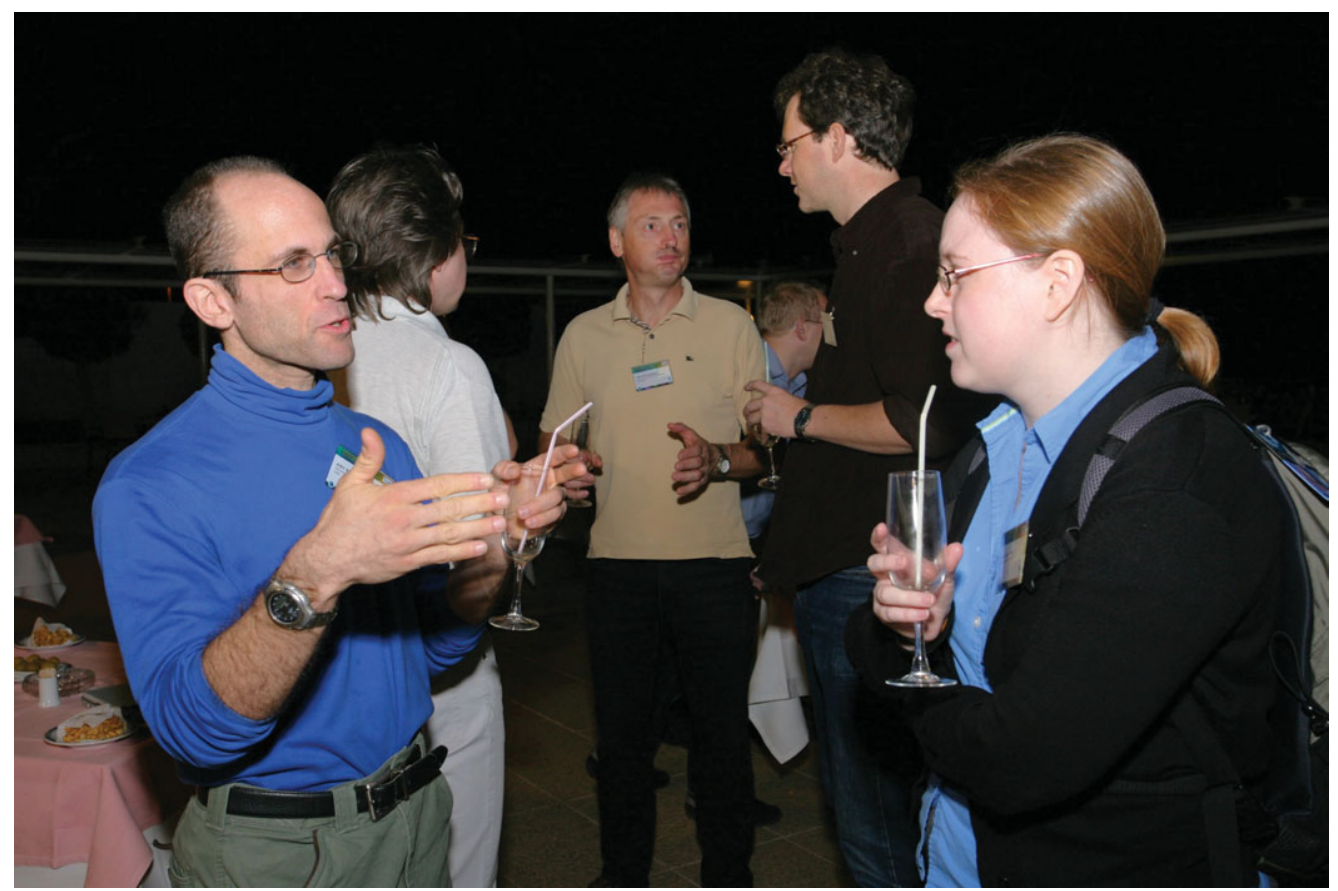

Eric Blackman talking to Amanda Kepley. In the background: M. Khodachenko, U. Motschmann and A. Reiners (from left to right)

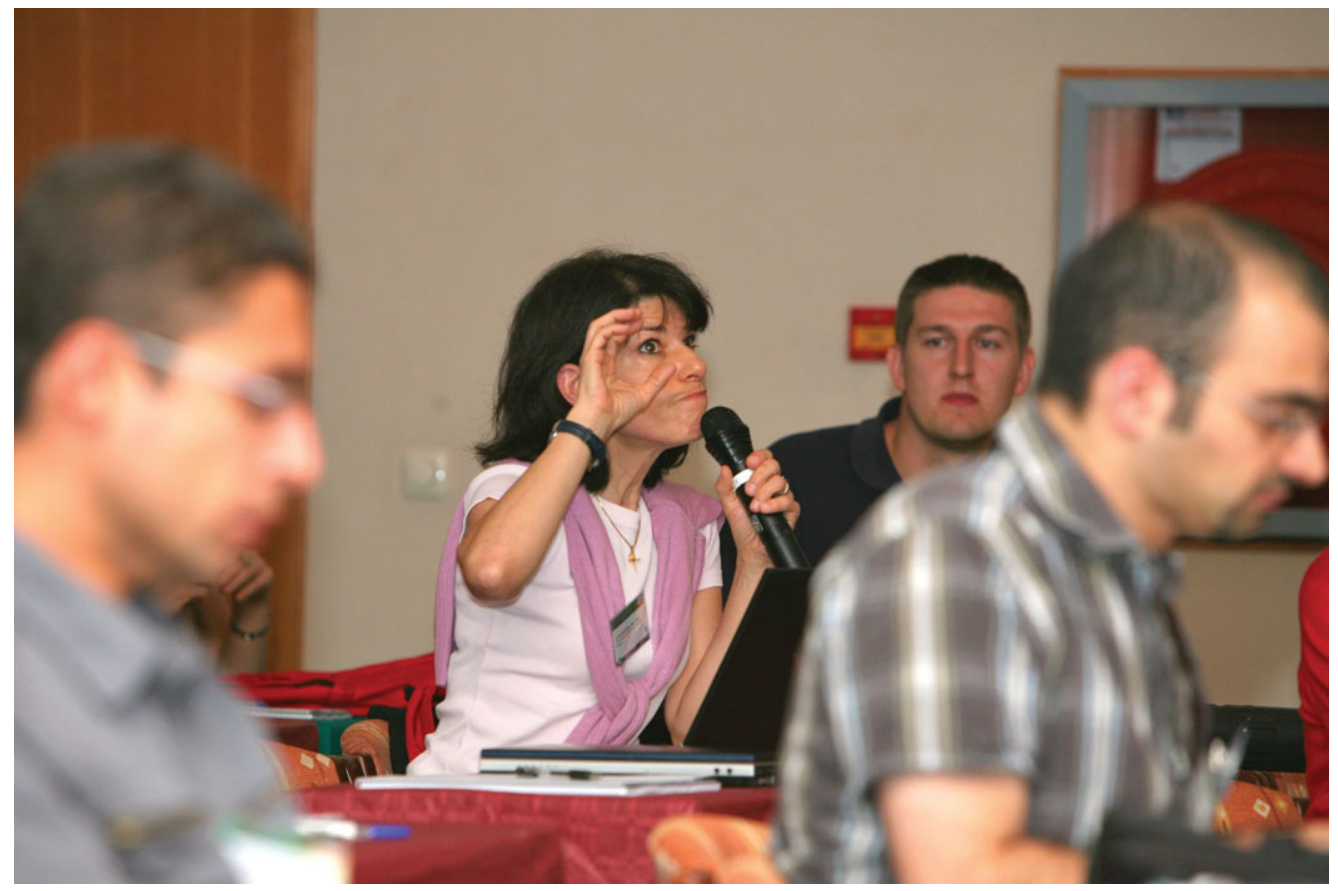

Elisabete de Gouveia dal Pino asking, Christoph Küker (in the background) listening. 